\title{
Die Bedürfnisse des Patienten in den Vordergrund stellen
}

Der diesjährige Galderma Ästhetiktag bot Fachvorträge, Live-Demonstrationen von ästhetischen Behandlungen sowie einzigartige Einblicke in die Gesichtsanatomie. Ein Schwerpunkt war neben dem Konzept der systematischen Gesichtsanalyse auch die auf die individuellen Patientenbedürfnisse ausgerichtete Produktwahl.

Durch die globale demografische Entwicklung verändern sich stetig die Anforderungen an die nicht invasive ästhetische Medizin. „Bis 2020 werden voraussichtlich eine Milliarde Menschen über 60 Jahre alt sein und sich aufgrund des natürlichen Hautalterungsprozesses mit einer Verschlechterung der Haut konfrontiert sehen“, sagte Marion Bock, Geschäftsführerin von Galderma.

\section{Ästhetische Medizin unterliegt ständigem Wandel}

Immer mehr Menschen äußern den Wunsch nach einer gesunden Haut und natürlichen Ausstrahlung, ohne dabei die eigene Persönlichkeit zu verändern. „Die nicht invasive ästhetische Medizin unterliegt einem ständigen Wandel. Ziel ist es, daraus Chancen abzuleiten, um kontinuierlich eine hohe Patientenzufriedenheit zu erreichen", so Dr. Alexandra Ogilvie, Fachärztin für Dermatologie und Allergologie in München. Sie führte zusammen mit Dr. Said Hilton, Facharzt für Dermatologie, Allergologie und Venerologie in Düsseldorf, durch das ganztägige Symposium.

Eine zunehmende Bedeutung gewinnt der Wunsch der Patienten nach individu- ellen ästhetischen Behandlungsprogrammen. Vor diesem Hintergrund wurde von internationalen Experten das Konzept der systematischen, standardisierten Gesichtsanalyse entwickelt. Es unterstützt den Arzt dabei, seine Patienten auf Basis genauer Kenntnisse der Gesichtsanatomie individuell einzuordnen.

\section{Auswahl aus vier \\ Produktkategorien}

Darauf aufbauend kann über die vier Produktkategorien Relax, Restore, Enhance und Refresh die Wahl des bestgeeigneten Produkts erfolgen. So kann bei jeden Patienten ein zufriedenstellendes Ergebnis erreicht werden. Im Rahmen des Ästhetiktags 2017 wurde das Konzept der systematischen Gesichtsanalyse von den nationalen und internationalen Referenten anhand von Live-Behandlungen präsentiert und mit den Symposiumsteilnehmern diskutiert.

Gudrun Girrbach

Galderma Ästhetiktag 2017; Düsseldorf, 20. Mai 2017; Veranstalter: Galderma Laboratorium

\section{Die neue Dimension der fraktionalen RF}

Lutronic, Hersteller hochentwickelter Lasertechnologien für ästhetische und medizinische Anwendungen, eröffnete vor Kurzem seine erste Niederlassung in Deutschland. Seit April 2017 ist das Unternehmen im hessischen Rödermark zu finden. Mit seinem Produktangebot deckt die Firma nahezu das gesamte Spektrum nicht invasiver Behandlungen ab. Ein Highlight des Unternehmens ist Infini $^{\text {Tx }}$ : ein fraktionales RF-System zur Behandlung jedes Hauttyps, das optimale Ergebnisse liefert.

\section{Microneedle fractional RF (MFR)}

Die „flow needling technology ${ }^{\text {тm c }}$ ermöglicht das exakte Erreichen der zu behandelnden Hautschichten. Eine neu entwickelte Software vereinfacht dabei die Handhabung und optimiert die Energieabgabe mit optimalen Behandlungsergebnissen für die Patienten. 49 feinste, vergoldete Nadeln dringen in die Haut ein, sodass punktförmige Mikroverletzungen des Hautgewebes entstehen. Gleichzeitig erfolgt über die Spitzen der
Mikronadeln ein Austausch von Energie. Als Reaktion wird die Reparaturfunktion der Haut ausgelöst, in deren Folge vermehrt Kollagen, Elastin sowie Hyaluronsäure ausgeschüttet wird.

Mit Infini ${ }^{\text {TM }}$ können folgende Indikationen effektiv behandelt werden: Hautalterung im Gesicht, Mimikfalten, Hautlaxheit, Narben, Aknenarben, übermäßige Talgproduktion und grobporige Haut sowie Dehnungsstreifen.

Primäres Ziel der Deutschland-Niederlassung ist es, bestehenden und künftigen Kunden einen noch besseren und schnelleren Service zu bieten. Umfangreicher Support - dazu gehören ausführliche Einweisungen, Schulungen und spezielle Ausbildungsprogramme für Ärzte - ist für Lutronic ein entscheidendes Anliegen.

Ralf Schmidt, National Sales Director der Lutronic Medical Systems Germany $\mathrm{GmbH}$, sieht den Aufbau des ersten Standortes eines koreanischen Unternehmens in diesem speziellen medizinischen Bereich als spannende Herausforderung:
„Mein Ziel ist es, Lutronic in Deutschland zu einem verlässlichen Partner für Ärzte und Kliniken aufzubauen und damit eine solide Basis für den europäischen Markt zu installieren. Der direkte Kundenservice und -support hat für mich persönlich einen besonderen Stellenwert.“

Nach Informationen von Lutronic

Das fraktionale RF-System eignet sich zur Behandlung jedes Hauttyps.

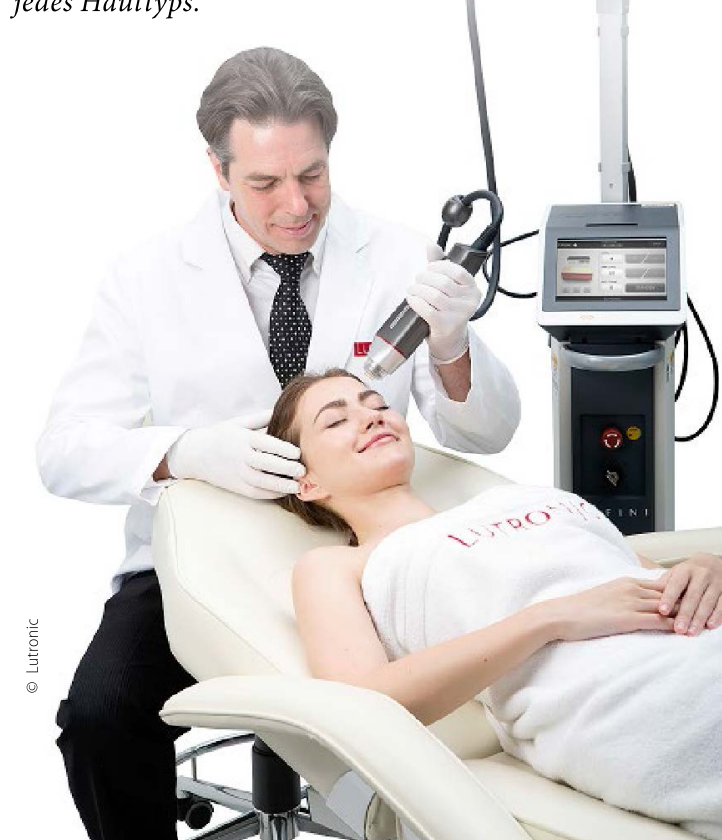

